\title{
Serendipitous detection of galaxies behind the Milky Way from the DENIS survey ${ }^{\star, \star \star}$
}

\author{
I. Vauglin ${ }^{1}$, J. Rousseau ${ }^{1}$, G. Paturel ${ }^{1}$, J. Borsenberger ${ }^{4}$, N. Epchtein ${ }^{2}$, P. Fouqué ${ }^{5}$, S. Kimeswenger ${ }^{6}$, \\ T. Le Bertre ${ }^{7}$, and G. A. Mamon ${ }^{4,3}$ \\ 1 CRAL-Observatoire de Lyon, 69561 Saint-Genis Laval Cedex, France \\ 2 Observatoire de la Côte d'Azur, Dept. Fresnel, 06304 Nice Cedex, France \\ 3 Observatoire de Paris-Meudon, 5 place Jules-Janssen, 92195 Meudon, France \\ 4 Institut d'Astrophysique de Paris, 98 bis Bld Arago, 75014 Paris, France \\ 5 European Southern Observatory, Casilla 19001, Santiago 19, Chile \\ 6 Institut für Astronomie, Innsbruck University, 6020 Innsbruck, Austria \\ 7 Observatoire de Paris, 61 Av. de l'Observatoire, 75014 Paris, France
}

Received 27 June 2001 / Accepted 31 January 2002

\begin{abstract}
A search has been undertaken at Lyon Observatory to identify, by eye, galaxy candidates at galactic latitudes lower than \pm 15 degrees on the DENIS $J$ and $K$-band images. This paper presents a list of 2028 objects that were serendipitously detected throughout the DENIS survey. Cross-identification with galaxy entries in the LEDA Database has been performed. Comparison with the second release of the 2MASS survey led to a satisfactory agreement of $J$ band magnitudes (std. dev. $=0.3 \mathrm{mag}$ ). The distribution of galaxy candidates along the Galactic Plane shows a concentration near the galactic longitude $l=305$ deg. As a by product of this inspection of $J$ and $K$ images some interesting galactic objects were found (star clusters and nebulae).
\end{abstract}

Key words. galaxies: general - infrared: galaxies - large-scale structure of Universe- catalogs - surveys dust, extinction

\section{Introduction}

The goal of the Deep Near Infrared Survey of the Southern Sky (hereafter DENIS) is to survey the entire Southern sky simultaneously in three wavelength bands (Gunn-i: $0.80 \mu \mathrm{m}, J: 1.25 \mu \mathrm{m}$ and $K \mathrm{~s}: 2.15 \mu \mathrm{m}$ ) with limiting magnitudes $18.5,16.5$ and 14.0 respectively. The observations were performed with the ESO 1 m-telescope at La Silla (Chile) with a dedicated camera. A detailed description of DENIS is given in Epchtein (1997). DENIS operations ended in early September 2001 and cover $97 \%$ of the sky. Data processing and archiving are still going on, public access to the data has begun. The data analysis is under way.

The results presented in this paper are based on the visual examination of uncalibrated DENIS $J$ and $K_{\mathrm{s}}$-band images located at galactic latitudes $|b|<15 \mathrm{deg}$. This is only a portion of the galactic plane and images are

Send offprint requests to: I. Vauglin, e-mail: vauglin@obs.univ-lyon1.fr

* Based on observations collected at the European Southern Observatory, La Silla, Chile.

** The catalog (Table 1) is only available in electronic form at the CDS via anonymous ftp to cdsarc.u-strasbg.fr (130.79.188.5) or via

http://cdsweb.u-strasbg.fr/cgi-bin/qcat?J/A+A/387/1 used as they become available, without insuring systematic coverage.

For galaxy recognition at these latitudes, visual inspection of the images is definitely better than automatic programme because, although automatic galaxy recognition programme work reasonably well at high latitudes, they are much less efficient in the crowded fields of the galactic plane. Automated products in this zone are polluted by a large fraction of false extended objects.

Besides this work, a systematic detection and extraction of extragalactic sources on the $I$-band DENIS images is also performed and has been presented in Vauglin et al. (1999).

One of the great interests of near infrared (NIR) surveys such as DENIS and 2MASS is that the low extinction in the NIR allows one to probe the local Universe behind the Milky Way. Compared to $V$, the extinction is 3.5 times lower in $J$ and 9 times lower in $K_{\mathrm{s}}$.

Our Milky Way hides nearly $25 \%$ of the entire sky. The systematic search of galaxies behind the Milky Way, which hides among others the region near the putative Great Attractor (Dressler et al. 1987), is of significant interest for studies requiring homogeneous samples, such as the kinematics of the local universe, the distance scale, large scale clustering, etc. 
Section 2 gives a brief description of observation and processing of $J$ and $K_{\mathrm{s}}$ DENIS images. The list of objects from these images is given in Sect. 3. In Sect. 4, we describe the cross-identification with 2MASS galaxies leading to the comparison of astrophysical parameters. Finally, in Sect. 5 we describe the distribution of galaxies as seen from this preliminary study and conclude about the future strategy.

\section{Observation and method}

The data processing procedure is described by Rousseau et al. (2000). In the present analysis, each image $^{1}$ is checked by eye to detect non-stellar objects. As in most extragalactic catalogs, the visual detection is based first on the extended nebular aspect of the sources. The detection of every object was then confirmed on the ESO- $R$ plates. Then, objects without counterpart on the $K$-band DENIS images or with high central peak were rejected because they could have been faint stars. The cross-identification with previous extragalactic catalogs, when overlapping, gives us confidence in the extragalactic nature of a number of our galaxy candidates. Patchy objects were also rejected because they might be star clusters rather than galaxies. We think that noise peaks do not contaminate our sample because they are easy to reject: they are very intense peaks almost without surrounding extension.

The external diameter is measured on the $J$ frame with no fixed limiting surface brightness. Nevertheless, we estimated the mean surface brightness approximately to $23.4 \pm 1.7 \mathrm{magJ} / \operatorname{arcsec}^{2}$. A circle is visually adjusted to fit the major axis and a smaller circle to fit the minor axis. This provides an estimation of the major and minor axes. The magnitude is calculated through a circular aperture encompassing the total extent of the galaxy. The sky background is substracted and superimposed stars are removed manually. The position angle is calculated through a visual adjustement of the direction of the major axis. Because of the short exposure times the position angle corresponds to the orientation of the inner region of each galaxy.

The preliminary astrometric calibration was not available at the time we processed these images. So, each galaxy coordinates were measured using the Digitized Sky Survey (DSS) following the procedure previously used (Paturel et al. 1999). The standard deviation of the coordinates measured on the DSS, for galaxies, is typically of a few arcsec. This will be discussed in Sect. 4 .

\section{The list of galaxies}

An internal cross-identification has been made and objects measured twice (or more) are combined using a simple averaging of magnitudes and coordinates. This allows us to determine an internal standard deviation for magnitudes.

\footnotetext{
1 After the first processing done at the PDAC (dark and bias substraction, glitches removal, flat fielding).
}

Note that some galaxies are not detected in $J$-band, while they are in $K$-band. Table 1 gives the first galaxies of the list extracted from this visual inspection. The entire table is available in electronic form through the CDS. This sample list is built to show different occurrences of comments and names. The arrangement of columns is as follows: Column 1: Right ascension and declination for epoch J2000.

Column 2: $J$ magnitude (possibly with an uncertainty $\operatorname{mark}(:))$.

Column 3: Mean error on $J$ calculated from multiple measurements on different frames.

Column 4: $J-K$ color estimate.

Column 5: Apparent diameter visually measured on the $J$ frame (in log of $\operatorname{arcsec}$ ).

Column 6: Major to minor axis ratio in logarithmic scale. Column 7: Position angle measured from North towards East (in degrees).

Column 8: Morphological type: S for spirals, SB for barred spirals, E for Elliptical, M for multiple or interacting, A for strong absorption, LSB for low surface brightness, ? or ?? for questionable identification.

Column 9: Alternate names from various catalogues: PGC (Leda@obs.univ-lyon1.fr; Paturel et al. 1989; Paturel et al. 2000), ESO (Lauberts \& Valentijn 1989), IRAS, CGMW (Saito), RKK (Kraan-Korteweg), WKK (Woudt et al.) and ZOAG (Saurer et al. 1997; Seeberger et al. 1996, 1998; Weinberger et al. 1980, 1999).

We checked that these objects are not already known as Globular clusters (Harris \& Racine 1979), Open clusters (Lyngå 1983), Bright Nebulae (Lynds 1965), HII regions (Sharpless 1959), or Planetary Nebulae (Acker et al. 1992). Note that the coordinates of planetary nebulae were re-measured by two of us (JR and GP). The list of these improved coordinates was sent to the CDS. 87 galaxy candidates are close to such galactic objects but only two objects coincide exactly with Planetary Nebulae. They are given at the end of Table 1.

Moreover, we cross-identified this list with those made by teams currently searching galaxies near the galactic plane (Weinberger 1980; Saito et al. 1990; Saito et al. 1991; Roman et al. 1996; Roman et al. 1998; Seeberger et al. 1996; Lercher et al. 1996; Saurer et al. 1997; Seeberger \& Saurer 1998; Kraan-Korteweg et al. 1995; Woudt et al. 1999; Weinberger et al. 1999; Kraan-Korteweg 2000).

Finally, we checked extended objects in the second release of the 2MASS survey. This is described in the next section.

We can suspect some biases in our sample. The relative number of elliptical galaxies versus spiral increases near the Galactic Plane (47\% of ellipticals at $|b|<7.5 \mathrm{deg}$ and $26 \%$ for $7.5<|b|<15 \mathrm{deg})$. Thus, we probably classified some spiral galaxies as ellipticals near the Galactic Plane because the spiral structure is lost when extinction increases. Yet, nearly edge-on galaxies are easier to select thanks to their non ambiguous form. Further, because of our relatively high detection threshold, the LSB galaxies are misrepresented in the present catalog (only $0.3 \%$ ). 
Table 1. Preliminary list of 2026 galaxies (and 2 PN's) visually detected in the region $|b|<15$ deg. The full table is available in electronic form at the CDS.

\begin{tabular}{|c|c|c|c|c|c|c|c|c|}
\hline $\begin{array}{r}\text { RA2000 DEC2000 } \\
\text { hms }^{\circ}, "\end{array}$ & $\begin{array}{r}J \\
\text { mag }\end{array}$ & $\begin{array}{l}\text { m.e. } \\
\text { mag }\end{array}$ & $\begin{array}{r}J-K \\
\text { mag }\end{array}$ & $\begin{array}{r}\log D \\
\mathrm{D} \text { in }\end{array}$ & $\overline{\log R}$ & $\begin{array}{l}\mathrm{PA} \\
\mathrm{deg}\end{array}$ & Type & Alternate Name \\
\hline J072944.1-103208 & & & & 0.81 & $\overline{0.19}$ & 41. & $\mathrm{E}$ & \\
\hline J072949.6-253457 & & & & 0.73 & & & $\mathrm{~S}$ & PGC0077391 CGMW2-916 ZOAG239.92-3.61 \\
\hline J082417.2-231156 & 14.6 & 0.3 & & 0.56 & & & & PGC0081139 CGMW2-3429 \\
\hline J082432.1-134653 & 13.7 & 0.3 & & 0.75 & & & & PGC0933319 \\
\hline J082436.3-270715 & 15.9 & 0.3 & & 0.45 & & & & \\
\hline J082449.6-135134 & 13.0 & 0.3 & 0.02 & 0.91 & 0.16 & 51. & & \\
\hline J082452.9-243010 & 15.8 & 0.3 & & 0.45 & & & & \\
\hline J083608.1-185431 & & & & 0.92 & & & & PGC0024172 ESO562-21 \\
\hline J083656.6-650117 & 14.1 & 0.3 & & 0.86 & 0.35 & 153. & $\mathrm{~S} ?$ & \\
\hline J083815.6-262812 & & & & 0.93 & 0.43 & 118. & $\mathrm{~S}$ & PGC0081707 CGMW2-4045 \\
\hline J083815.8-274945 & & & & 0.86 & & & M & PGC0086235 CGMW2-4048 \\
\hline J083820.9-185826 & & & & 0.75 & 0.21 & 43. & $\mathrm{E} ?$ & PGC0180997 \\
\hline J083822.2-263014 & & & & 0.78 & & & & \\
\hline J093215.9-651321 & 15.0 & 0.3 & & 0.66 & & & & PGC2791849 RKK1443 \\
\hline J100724.7-630440 & 14.0 & & & 0.91 & 0.25 & 11. & $\mathrm{~A}$ & \\
\hline J100724.7-630440 & 14.0 & & 1.05 & 0.82 & & & & \\
\hline J100726.0-650249 & 14.6 & & 0.68 & 0.78 & & & $\mathrm{~S} \mathrm{~A}$ & PGC2792231 RKK1973 \\
\hline $\mathrm{J} 101118.9-410816$ & 13.1 & 0.3 & & 0.97 & 0.24 & 43. & & PGC0029674 ESO316-45 IRAS10092-4053 \\
\hline J101131.6-395159 & 13.7 & 0.3 & & 0.93 & & & $?$ & PGC0593140 \\
\hline J101458.4-622221 & 15.1 & 0.3 & & 0.41 & & & $? ?$ & PGC2792355 RKK2154 \\
\hline J103755.2-712327 & 13.6 & 0.3 & 1.41 & 0.98 & 0.40 & 138. & SB & PGC0269167 \\
\hline J104708.8-651747 & 14.5 & 0.3 & 0.29 & 0.76 & 0.42 & 42. & $\mathrm{~S} \mathrm{~A}$ & \\
\hline J121250.2-501435 & 12.2 & 0.2 & & 1.10 & 0.27 & 131. & $\mathrm{E}+\mathrm{S}$ & \\
\hline J122916.0-544509 & 14.2 & 0.2 & & 1.04 & 0.54 & 167. & $\mathrm{~S} \mathrm{M}$ & \\
\hline J122947.6-562221 & 15.7 & 0.3 & & 0.53 & & & & \\
\hline J130352.5-580124 & 12.5 & 0.2 & 1.06 & 0.96 & 0.36 & 15. & S star & PGC0382951 \\
\hline $\mathrm{J} 141037.2-520607$ & 11.9 & 0.3 & 0.67 & 1.11 & & & $\mathrm{E}$ & PGC0448694 WKK3892 \\
\hline J160923.0-303008 & & & & 0.99 & & & LSB & \\
\hline J170139.9-235520 & 12.7 & 0.3 & 0.60 & 0.88 & 0.20 & 40. & $\mathrm{E} ?$ & \\
\hline J161041.1-545735 & & & & 0.86 & & & $\mathrm{PN}$ & \\
\hline J175936.4-463854 & 11.7 & 0.3 & 0.65 & 1.21 & 0.32 & 29. & $\mathrm{PN}$ & PGC0061236 ESO279-14 \\
\hline
\end{tabular}

\section{Cross-identification with the 2MASS survey}

It is very difficult to estimate how complete is our list. The region covered in this serendipitous inspection represents 16260 frames among 198720 for a complete coverage of this galactic band $(|b|<15 \mathrm{deg})$, i.e. $8 \%$. This means that a full coverage would have given 25000 galaxy candidates. For comparison, 2MASS which covered about half of the southern sky detected 34017 extended objects in the same galactic band, i.e. 68034 extended objects for the full coverage. We conclude that 2MASS detects 2.6 times more objects. This means that 2MASS goes $2.6^{1 / 3}=1.37$ deeper in distance (assuming that the number of objects increases as the cube of the distance) or $5 \log 1.37=0.7 \mathrm{mag}$ fainter. Actually, the difference is probably less than 0.7 mag because automatic search of 2MASS may be contaminated by false detection near the galactic plane.

We made the cross-identification with extended 2MASS objects by comparing the coordinates. Crossidentification is validated when there is only one 2MASS object matching our position within a radius of 15 arcsec. Among the 2026 galaxies listed in Table 1, 362 galaxies are also found in the 2MASS second release. We noted that this number does not increase significantly when increasing the radius of search up to 1 arcmin.

Thus, we have a rather small number of galaxies in common with 2MASS because the portion of the Galactic Plane studied here has probably few overlaps with the portion of the Galactic Plane already observed by 2MASS. Nevertheless, we want to use this common sample in order to compare the $J$-magnitude and the coordinates.

A comparison of $J$-band magnitudes is shown in Fig. 1. We used the 2MASS magnitude $j_{-} m$, an optimized aperture magnitude. There is a global agreement. A direct regression (we assume that the accuracy is better on $x$-axis for the 2MASS $J$-magnitudes because 2MASS probably reaches a fainter limit as said above and thus works with better $S / N$ ratio) shows that the slope is not significantly different from one but that the zero points are different. After rejection of 33 discrepant points at the $2.5 \sigma$ level, the slope is found to be $1.04 \pm 0.02$ with 254 points. This value is not significantly different from one (the $t$ of the Student $t$-test is 2.0, i.e. slightly below the theoretical value 2.1 at the 0.01 probability level). Forcing a slope of one, the zero point is found to be $0.28 \pm 0.02$. This value is significantly 


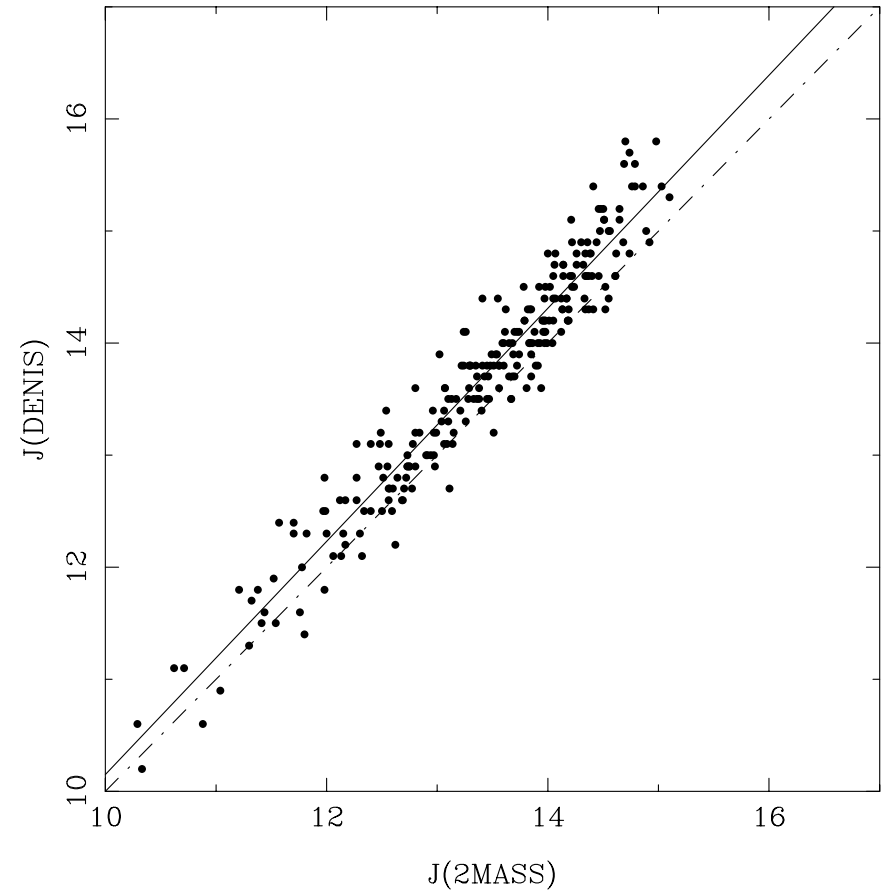

Fig. 1. Comparison of $J$ band magnitudes from DENIS and 2MASS surveys. The dashed line is the first bissect. The solid line is the direct regression line.

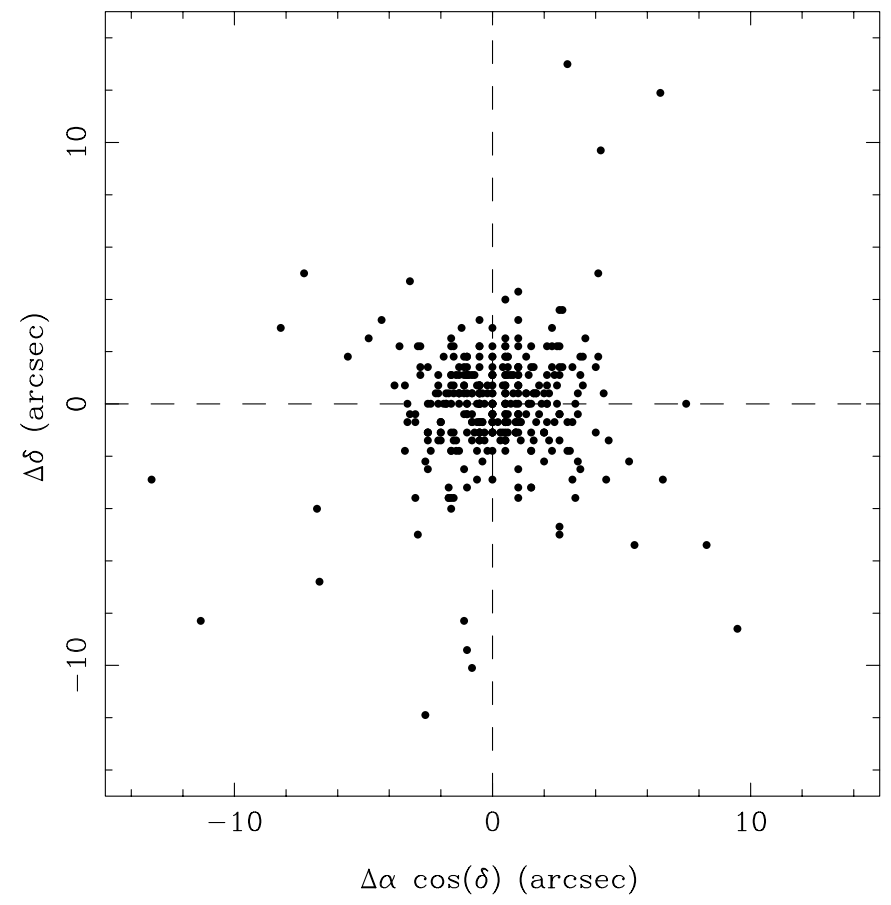

Fig. 2. Comparison of coordinates from DENIS and 2MASS surveys.

different from zero. This is obvious in Fig. 1 where the unit slope is drawn (dashed line). The standard deviation of the regression is $\sigma=0.29$. To estimate the flux of our galaxy candidates, we have removed superimposed stars, when occurring. This can partly explain the difference between 2MASS and DENIS $J$-magnitudes, which are 0.28 higher. Besides, our magnitudes are not pure asymptotic

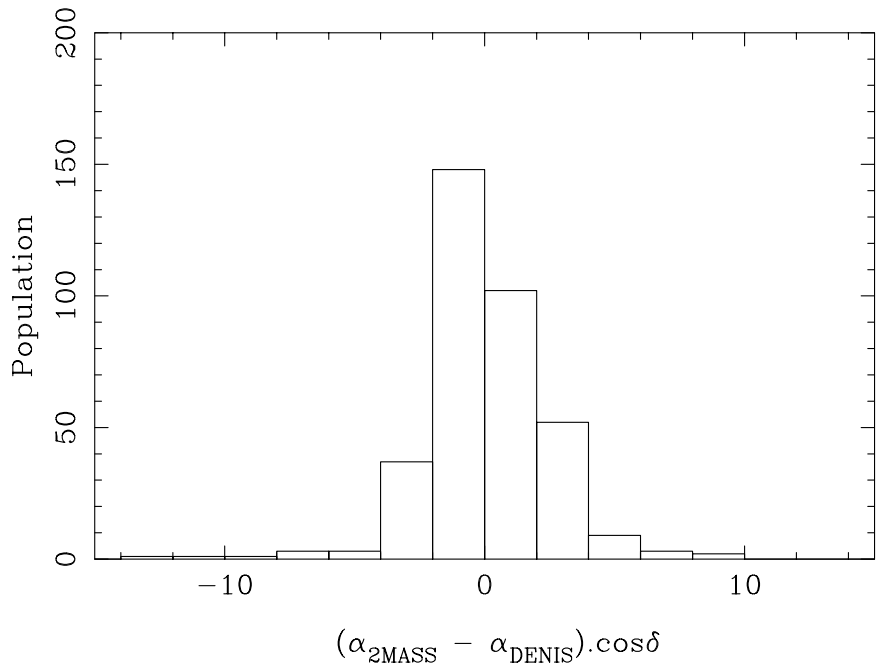

Fig. 3. Histogram of the deviation $(\alpha(2 M A S S)-$ $\alpha(D E N I S)) \cos \delta$.

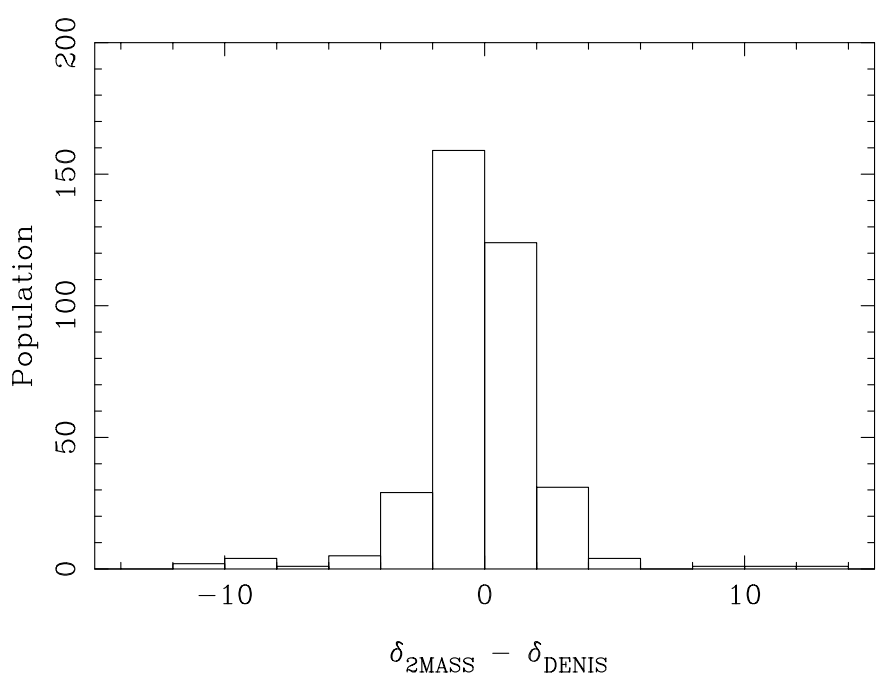

Fig. 4. Histogram of the deviation $(\delta(2 M A S S)-\delta(D E N I S))$.

magnitudes. This also can explain a part of the zero-point difference with 2MASS.

A comparison is made between our coordinates and 2MASS coordinates. Because we performed the crossidentification within a circle of 15 arcsec, we cannot expect an error larger than this value. Nevertheless, one should find a much smaller dispersion to confirm our cross-identifications. Figures 2 to 4 exhibit the comparison. The agreement is satisfactory. The standard deviation is 2.3 arcsec in Right Ascension and 2.2 arcsec in Declination. This means that the cross-identification can be made simply by comparing coordinates.

This list gives 2028 objects (presumably galaxies except for two identified planetary nebulae). For each object we give a visual estimate of the major and minor axis diameter and of the position angle. For 1863 objects a measure of the $J$ magnitude is given. Among these 2028 objects 1048 are presently uncatalogued but 362 are detected by the 2MASS survey (second release). A $J-K$ color 


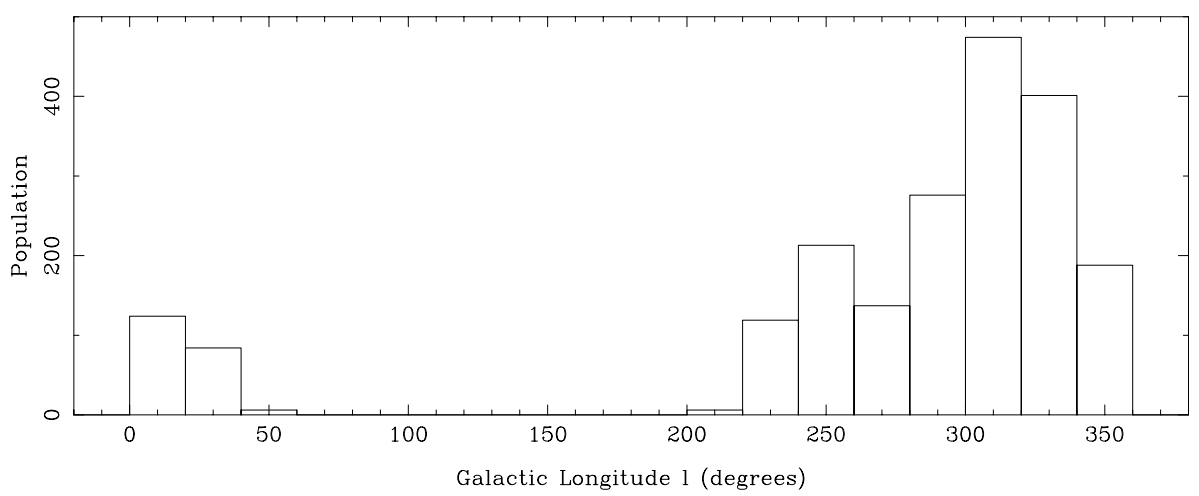

Fig. 5. Distribution of the sample in galactic longitude. Most of the objects are located between $l=200 \mathrm{deg}$ and $l=350 \mathrm{deg}$.

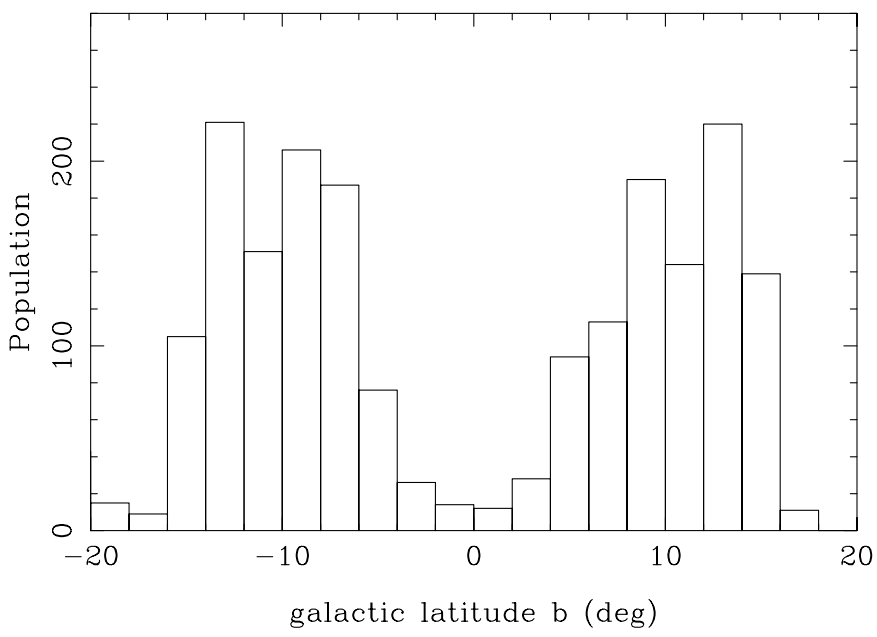

Fig. 6. Distribution of the sample in galactic latitude. Most of the objects are located in the range $b= \pm 15 \mathrm{deg}$. The depression near $b=0$ is due to the galactic extinction.

estimate is also given for 735 objects. It is to be noted that the apertures used to determine $J$ and $K$ magnitudes are not exactly the same. The color must be considered as tentative only. Some objects are too faint in $J$ to be measured. They are simply detected in $K$.

\section{Distribution of galaxies}

We are currently limited to a very partial coverage of the Zone of Avoidance. Thus, the interpretation of the largescale galaxy distribution is not possible. Nevertheless it appears that many galaxies are located near the putative Great Attractor $\left(l=307^{\circ} ; b=9^{\circ}\right.$ according to Dressler et al. 1987) and in a wide region around. The distribution in galactic longitude (Fig. 5) shows a maximum at $l=305 \mathrm{deg}$. The Flamsteed equal area projection in supergalactic coordinates (Fig. 7) shows how the strips extend accross the galactic band $|b|<15 \mathrm{deg}$. Taking into account that we extended the domain slightly outside the range $|b|<15 \mathrm{deg}$, especially in the region where we found many galaxies, the bump is significant (the peak should simply be reduced by about 50 objects).
The distribution of these galaxies in galactic latitude is shown in Fig. 6. This figure is important for the definition of the future (presently ongoing) strategy. It appears from this figure that the number of detections drops dramatically for $|b|<5 \mathrm{deg}$. In this region one cannot expect many detections in $I$-band and one should continue the visual detection from the $J$ and $K$-band frames. On the other hand, by checking our objects on the ESO $R$-band photographic plates it appears that almost all galaxies above $|b|=5 \mathrm{deg}$ are visible. This leads us to conclude that the systematic search in the region $5 \mathrm{deg}<|b|<10 \mathrm{deg}$ should be made with the $I$-band frames by a visual inspection. Above $|b|=10 \mathrm{deg}$ we will use the automatic detection in $I$-band. As a test we will use an intermediate region where both visual and automatic methods will overlap.

\section{Conclusion}

The present list of $\approx 2000$ galaxies detected near the galactic plane $(|b| \leq 15 \mathrm{deg})$ by a visual inspection of $J$ and $K$ images gives secure detections in a region where automatic programs may lead to false detections. The listed objects were generally seen on both $J$ and $K$ frames but they were also checked on the $R$-band photographic plates. The conclusion of this inspection is that, in most cases, the galaxies are also visible in the $R$-band. This shows that a visual search in the region $5 \mathrm{deg}<|b|<10 \mathrm{deg}$ can be conducted successfully using $I$-band images. This work is now in progress.

Because the survey is almost automatic, it is very important to determine which images can be used for photometry. Indeed, without rejection of bad images, the number of discrepant measurements we got when comparing with an independent survey (2MASS) is relatively high $(11 \%)$. This can be explained by the use of nonphotometric nights ${ }^{2}$. In this respect, we suggested to systematically check the variations of the standard deviation of the sky background. We give an example in Fig. 8. Of course the deviation of the sky background may also be affected by crowded regions (and maybe by nearby bright stars). So, adjacent strips need to be compared as well.

\footnotetext{
2 These images are still valid for detection purposes.
} 


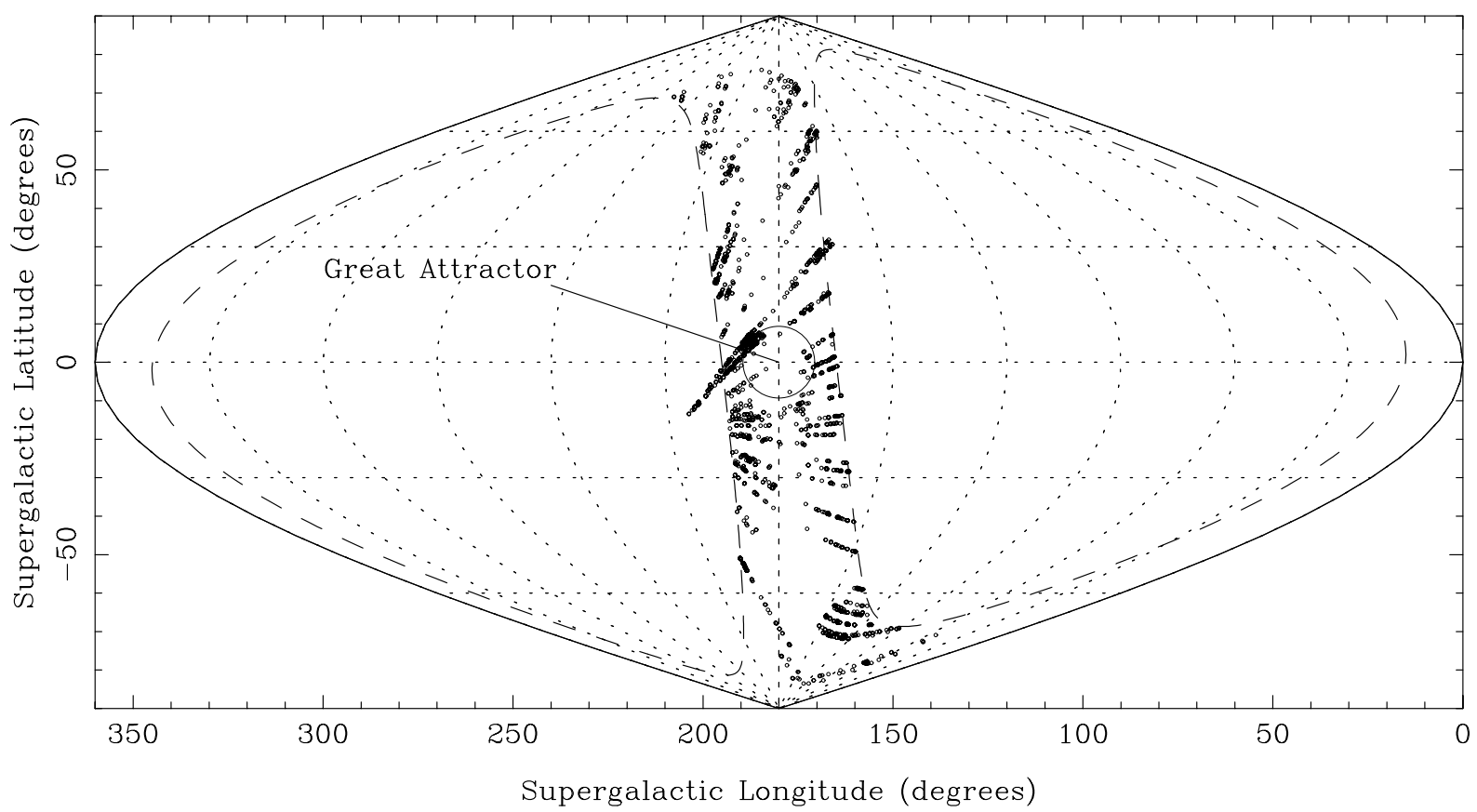

Fig. 7. Flamsteed equal area projection in supergalactic coordinates. There is a prominent concentration near the putative Great Attractor $\left(l=307^{\circ} ; b=9^{\circ}\right)$ but another concentration is present near the region studied in the previous paper (Rousseau et al. 2000). The galactic band in the southern hemisphere corresponds to the vertical band in the middle of the figure.

From this diagram it is easy to see which images are satisfactory for photometric purpose. Similarly, one can plot the typical size of a star image (FWHM) to detect poor seeing. A bad seeing is not a major drawback for photometry for an isolated object but can be a severe handicap in dense regions because it makes more difficult the removal of stars superimposed on galaxies.

Acknowledgements. We thank M. H. Lassalle, M. C. Marthinet and C. Petit for their technical assistance. The DENIS team is warmly thanked for making this work possible and in particular the Operations team at la Silla.

The DENIS program is partly funded by the European Commission through SCIENCE and Human Capital and Mobility grants. It is also supported in France by INSU, the Education Ministry and CNRS, in Germany by the Land of Baden-Würtenberg, in Spain by DGICYT, in Italy by CNR, in Austria by the Fonds zur Förderung der Wissenschaft und Forschung, in Brazil by FAPESP.

We made use of the ESO facility in Munich for retrieving images from the Digitized Sky Survey produced at the Space Telescope Science Institute under US Government grant NAG W-2166. It is based on photographic data obtained using The UK Schmidt Telescope and the Palomar Sky Survey.

The UK Schmidt Telescope was operated by the Royal Observatory Edinburgh, with funding from the UK Science and Engineering Research Council, until 1988 June, and thereafter by the Anglo-Australian Observatory. Original plate material is copyright (c) the Royal Observatory Edinburgh and the Anglo-Australian Observatory. The plates were processed into the present compressed digital form with their permission.



Fig. 8. A method to discriminate good and bad images. The plot of the standard deviation of the sky background along the 180 images of a strip shows when images are good for photometry. The upper curve is an example of a strip (MS9315) which starts to be good at the image number $\approx 100$ and which could be suspected to be non-photometric before. The lower curve is an example of a good strip (MS9319).

This publication makes use of data products from the Two Micron All Sky Survey, which is a joint project of the University of Massachusetts and the Infrared Processing and Analysis Center/California Institute of Technology, funded by the National Aeronautics and Space Administration and the National Science Foundation. 


\section{References}

Acker, A., Ochsenbein, F., Stenholm, B., et al. 1992, The Strasbourg-ESO Catalogue of Galactic Planetary Nebulae (Garching bei München, European Southern Observatory)

Danks, A. C., Wamsteker, W., Shaver, P. A., \& Retallack, D. S. 1984, A\&A, 132, 301

Dressler, A., Faber, S. M. \& Burstein, D., et al. 1987, ApJ, 313,37

Epchtein, N., de Batz, B., Capoani, L., et al. 1997, The Messenger, 87, 27

Harris, W. E., \& Racine, R. 1979, ARA\&A, 17, 241

Kraan-Korteweg, R. 2000, A\&AS, 141, 123

Kraan-Korteweg, R., Fairall, A. P., \& Balkowski, C. 1995, A\&A, 297, 617

Lauberts, A., \& Valentijn, E. A. 1989, The Surface Photometry Catalog of the ESO-Uppsala Galaxies (ESO, Munich)

Lercher, G., Kerber, F., \& Weinberger, D. H. 1996, A\&AS, 117, 369

Lynds, B. T. 1965, ApJS, 12, 163

Lyngå, G. 1983, Revised Catalogue of Open Cluster Data, Lund Observatory

Paturel, G., Fouqué, P., Bottinelli, L., \& Gouguenheim, L. 1989, A\&AS, 80, 299
Paturel, G., Petit, C., Garnier, R., \& Prugniel, P. 1999, A\&AS, 140,89

Paturel, G., Fang, Y., Petit, C., Garnier, R., \& Rousseau, J. 2000, A\&AS, 146, 19

Roman, A. T., Nakanishi, K., Tomita, A., et al. 1996, PASJ, 48,679

Roman, A. T., \& Saito, M. 1998, Contrib. Dept. Astro. Kyoto Univ., 570

Rousseau, J., Paturel, G., Vauglin, I., et al. 2000, A\&A, 363, 62

Saito, M., Ohtani, H., Asonuma, A., et al. 1990, PASJ, 42, 603

Saito, M., Ohtani, H., Baba, A., et al. 1991, PASJ, 43, 449

Saurer, W., Seeberger, R., \& Weinberger, D. H. 1997, A\&AS, 126, 247

Seeberger, R., Saurer, W., \& Weinberger, R. 1996, A\&AS, 117,1

Seeberger, R., \& Saurer, W. 1998, A\&AS, 127, 101

Sharpless, S. 1959, ApJS, 4, 257

Vauglin, I., Paturel, G., Borsenberger, J., et al. 1999, A\&AS, 135,133

Weinberger, R., Gajdosik, M., \& Zanin, C. 1999, A\&AS, 137, 293

Weinberger, R. 1980, A\&AS, 40, 123

Woudt, P. A., Kraan-Korteweg, R., \& Fairall, A. P. 1999, A\&A, 352,39 